\title{
Never too old for an inherited condition
}

\author{
P Robinson \\ PCD Diagnostic service \\ Department of Respiratory and Sleep Medicine, \\ Royal Children's Hospital, \\ Murdoch Children's Research Institute and \\ Department of Paediatrics, University of Melbourne.
}

\section{Corresponding Author}

\author{
Assoc Prof Phil Robinson \\ Dept Respiratory and Sleep Medicine \\ Royal Children's Hospital, \\ Parkville 3052 \\ Phil.robinson@rch.org.au \\ Ph: 0393455818
}

This is the author manuscript accepted for publication and has undergone full peer review but has not been through the copyediting, typesetting, pagination and proofreading process, which may lead to differences between this version and the Version of Record. Please cite this article as doi: $10.1111 / \mathrm{imj} .14060$

This article is protected by copyright. All rights reserved. 


\begin{abstract}
:
Primary Ciliary Dyskinesia (PCD) is an uncommon, inherited condition causing progressive suppurative airway lung disease with subsequent bronchiectasis, chronic rhinitis-sinusitis, deafness and reduced fertility. Diagnosis is often delayed by lack of awareness of the condition in the medical community and limited access to the few centres in Australia able to do full diagnostic testing. This report details the late diagnosis of PCD in 2 adults who have had long standing interactions with medical services but in whom the diagnosis was never considered or even dismissed. Greater awareness of the condition will reduce time to diagnosis, prevent administration of ineffective therapy and allow earlier institution of targeted therapy.
\end{abstract}

\title{
Introduction:
}

Primary Ciliary Dyskinesia (PCD) is an autosomal recessively inherited condition often producing symptoms early in life resulting from structural and functional abnormalities of airway cilia. Diagnosis requires detailed assessment of these parameters in specialised diagnostic services in conjunction with genetic testing. Although suggestive symptoms may be present from early in life they are symptoms very common in childhood and unless specific features are considered the diagnosis may be missed. Late diagnosis in childhood is not uncommon in PCD however the recent introduction of a diagnostic service into the state of Victoria has highlighted that a significant number of adults in such a community may have PCD. Diagnosis in the adult patient requires a high level of suspicion and knowledge of strong suggestive features of PCD.

\section{History}

A 52 year old man was referred to the PCD diagnostic service at the Royal Children's Hospital, Melbourne after his long time treating respiratory physician suspected possible PCD after attending a lecture by the head of the diagnostic service. Following that lecture the treating physician had enquired about specific diagnostic clues and identified that the patient not only had long standing bronchiectasis but in addition had undergone a lobectomy at age 16. The patient was referred for diagnostic testing. Detailed questioning at the diagnostic service appointment revealed he had spent many periods in hospital as a child being treated for chest infections and had hearing issues as a child associated with recurrent otitis media. As an adult he had been married for over 20 years and had been unable to conceive naturally but had fathered two children with the aid of IVF. 
Sampling of nasal cilia revealed a dys-synchronous circling ciliary beat pattern suggestive of a central microtubular pair abnormality. This ultrastructural defect was confirmed on electron microscopy (Fig 1). At the diagnostic clinic consultation the patient mentioned that his elder brother, aged 72 years, had similar symptoms and history and was keen to investigate the possibility of him having the same diagnosis.

The elder brother was subsequently referred for diagnostic testing. He described multiple chest problems in childhood and regular admissions to hospital for treatment. At age 17 he underwent a right middle lobectomy for extensive bronchiectasis. He gave a history of recurrent otitis media as a child and had undergone grommet insertion twice in childhood. He described hearing problems from early childhood and had been fitted with hearing aids in primary school. He recalled participating in the state-wide TB X-ray screening program in the 1960's and being regularly recalled for assessment because of radiological changes on his Xray. Although cleared of TB on each occasion no explanation for these structural changes was ever provided. He had been married for many years but had been unable to conceive and tests had failed to identify a cause. He described a regular productive cough and was aware of his regular sniffing on an almost hourly basis for much of his adult life. In adult life he had undergone three extensive sinus surgeries with minimal improvement.

He had been seen regularly throughout his adult life by an adult respiratory physician but had never been advised to perform any form of airway clearance. The diagnosis of PCD had been raised by a physician at one stage but had been dismissed as he did not have dextrocardia. Following the mention of PCD he searched the internet and became convinced of his diagnosis. He was advised by his respiratory physician not to mention such a possibility to his brother so as not to worry him.

Nasal brushing was performed and under light microscopy clear circling cilial movement was apparent on plane view (from top). Electron microscopy examination revealed absent central pair microtubules identical to his brother.

In the first four years of the Victorian diagnostic service 286 patients have been referred for diagnostic testing and 31 cases of PCD identified (11.5\% positive testing). The average age of patients referred was 12.4 years, (11.2 years males 14.3 years females). The average age of patients diagnosed with PCD was 17.6 years, (males 18.7 years, females 16.7 years). There were 185 patients under the age of 18 referred for testing and 21 (11.3\% of tested) were found to have PCD. Of the 21 paediatric patients found to have PCD there were 14 males (average age 7.7 years) and 11 females (average age 10.7 years).

There were 63 patients over the age of 18 referred for testing and 10 (15.8\% of tested) were found to have PCD. Of the 10 adult patients found to have PCD there were 4 males (average age 46 years) and 6 females (average age 27.8 years). 


\section{Discussion}

Primary Ciliary Dyskinesia (PCD) is a progressive inherited condition resulting from structural and or functional defects in airway cilial function which leads to impaired airway muco-ciliary clearance and progressive lung damage culminating in bronchiectasis and upper airway issues including chronic sinusitis and hearing loss.

Diagnosis of PCD involves recognition of abnormal cilial beating on light microscopy, evidence of ultrastructural defects on electron microscopy or identification of two disease causing genes. Currently 36 genes have been identified as causing PCD however it is estimated that this only accounts for approximately $70 \%$ of all cases of PCD identified through all methods. Exhaled nasal nitric oxide has been shown to have a strong predictive value when levels of less than $77 \mathrm{ppb}$ are recorded.

The Victorian PCD diagnostic service was established in 2013. This service compliments the long standing diagnostic service that exists at the Concord Hospital in Sydney. Our diagnostic rate is very similar to that recorded from other services with similar referral practises. (L. Morgan Concord Hospital personal communication).

Our figures suggest that, at least in our Victorian community, PCD has historically been under diagnosed in the paediatric setting resulting in a significant number of adult patients being present in the community with regular symptoms of PCD but undiagnosed. It is likely in other communities without an easily accessible diagnostic service and general awareness of PCD in the medical community that similar late diagnoses are occurring, and additionally that some adult patients remain undiagnosed. The average age of diagnosis in our service at 17.6 years is significantly higher than that reported from longer standing diagnostic services where figures around 10 years are common. Increased awareness in the paediatric medical field is also important.

The diagnosis of PCD should be considered in adult patients who are being seen with suppurative lung disease, extensive upper airway suppurative disease and in infertility clinics. While these symptoms have multiple, and often far more frequent causes, several suggestive points on history and clinical examination may prompt the clinician to consider PCD and referral for diagnostic testing. An adult presenting with suggestive symptoms including a history of paediatric suppurative disease, such as described in the two cases of this present report, increases the possibility of PCD and would warrant referral for testing. The presence of laterality defects such as dextrocardia and total situs inversus should also raise the possibility of PCD however it is important to remember that less than $50 \%$ of patients found to have PCD will have laterality defects while only $20 \%$ of patients with dextrocardia will have PCD (1). In some populations consanguinity will increase the possibility of inherited diseases including PCD. O'Callaghan et al reported that in the Bradford PCD population, heavily of British Asian demographic, 52\% of patient's parents were first cousins (2) 
Leigh et al examined the clinical features of 205 paediatric (less than 18 years) patients found to have PCD from 534 patients referred for diagnostic testing. They found four criteriadefined clinical features were statistically predictive of PCD; laterality defects, unexplained neonatal respiratory distress, early-onset year-round nasal congestion and early onset yearround wet cough were strongly predictive of PCD (3). The specificity of a diagnosis of PCD when all 4 were present was $0.99,0.96$ when three were present and 0.72 when 2 were present. In adults however, such as in our described patients, the ability to obtain accurate information on 3 of these parameters (neonatal respiratory distress, early onset cough or upper airway congestion) is limited. Behan et al have developed a predictive score for patients referred with chronic daily productive cough. The PICADAR score comprises 7 predictive parameters; full term gestation, neonatal chest symptoms, neonatal intensive care admittance, chronic rhinitis, situs inversus and congenital heart defect (4). A specificity of 0.75 was obtained for a score of 5 points. Again determination of at least 3 of these parameters may be difficult in the adult populations and limits the usefulness of PICADRA score when assessing adult patients.

While PCD remains incurable the accurate diagnosis of patients with PCD has several important benefits. While many as yet undiagnosed PCD patients will be under treatment in Non CF bronchiectasis clinics recognition of the diagnosis of PCD may allow for institution of specific targeted therapy. While mutation specific therapy, such as has recently been developed in Cystic Fibrosis, is not yet available in PCD, specific therapy trials are ongoing attempting to address possible underlying inherent biochemical defects. (Clearing Lungs With ENaC Inhibition in Primary Ciliary Dyskinesia (CLEAN-PCD) ClinicalTrials.gov Identifier: NCT02871778)

Radiological studies of PCD consistently show that bronchiectasis in the upper lobes is much less common that that found in the middle and lower lobes. Performance of airway clearance techniques in the traditional seated position may in PCD not be ideal as this would optimally promote clearance from the upper less effected lobes. Identification of PCD in an adult may also be of use genetically in helping explain a patient's infertility.

In conclusion physicians treating adult patients with bronchiectasis should have a high index of suspicion of the diagnosis of PCD in the presence of laterality defects, a strong paediatric history of suppurative lung or upper airway disease and selective regional bronchiectasis with sparing of the upper lobes. When such clinical findings are present patients should be referred to diagnostic centres for specific testing. 


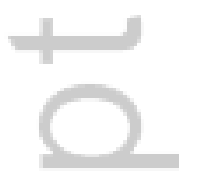

4 


\begin{abstract}
:
Primary Ciliary Dyskinesia (PCD) is an uncommon, inherited condition causing progressive suppurative airway lung disease with subsequent bronchiectasis, chronic rhinitis-sinusitis, deafness and reduced fertility. Diagnosis is often delayed by lack of awareness of the condition in the medical community and limited access to the few centres in Australia able to do full diagnostic testing. This report details the late diagnosis of PCD in 2 adults who have had long standing interactions with medical services but in whom the diagnosis was never considered or even dismissed. Greater awareness of the condition will reduce time to diagnosis, prevent administration of ineffective therapy and allow earlier institution of targeted therapy.
\end{abstract}


Never too old for an inherited condition

\author{
P Robinson \\ PCD Diagnostic service \\ Department of Respiratory and Sleep Medicine, \\ Royal Children's Hospital, \\ Murdoch Children's Research Institute and \\ Department of Paediatrics, University of Melbourne.
}

Corresponding Author

Assoc Prof Phil Robinson

Dept Respiratory and Sleep Medicine

Royal Children's Hospital,

Parkville 3052

Phil.robinson@rch.org.au

Ph: 0393455818

This article is protected by copyright. All rights reserved. 


\section{University Library}

\section{- M M I E E R VA A gateway to Melbourne's research publications}

Minerva Access is the Institutional Repository of The University of Melbourne

Author/s:

Robinson, $\mathrm{P}$

Title:

Never too old for an inherited condition

Date:

2018-10-01

Citation:

Robinson, P. (2018). Never too old for an inherited condition. INTERNAL MEDICINE JOURNAL, 48 (10), pp.1252-1254. https://doi.org/10.1111/imj.14060.

Persistent Link:

http://hdl.handle.net/11343/284553 